\title{
New salvage vincristine, carmustine, cyclophosphamide, and prednisone (VBCP) chemotherapy for relapsed/refractory multiple myeloma in the modern era: A case series
}

\author{
Abdullah M Khan¹, Jameel Muzaffar¹, Lazarus K Mramba² and Jan S Moreb,* \\ 1 Division of Hematology and Oncology, Department of Medicine, University of Florida, Gainesville, Florida, USA \\ 2 Statistician, Department of Medicine, University of Florida, Gainesville, Florida, USA
}

\begin{abstract}
We report our experience with vincristine, carmustine (BCNU), cyclophosphamide, and prednisone (VBCP), a regimen given in the outpatient setting to patients with multiple myeloma (MM) resistant to almost all the available novel/targeted therapies. Ten patients received salvage VBCP who were heavily pretreated with a median of 5.5 prior treatment regimens including lenalidomide, pomalidomide, bortezomib, carfilzomib, cyclophosphamide, and autologous stem cell transplantation. The objective response rate ( $\geq$ PR) was $60 \%$, with an additional $30 \%$ achieving stable disease (SD). The median time to progression for patients with $\geq$ SD was 3.6 months. The median progression free survival was 4.4 months while the median overall survival was 12.8 months. Despite severe myelosuppression being the main toxicity, $61 \%$ of the subsequent cycles were given on time. Treatment-related mortality was not observed. Our results suggest VBCP is a highly active and tolerable salvage regimen among heavily pretreated MM patients who already failed many of the novel agents.
\end{abstract}

Keywords: multiple myeloma; novel agents; salvage therapy; BCNU; autologous stem cell transplantation

\section{Introduction}

Over 30,000 new cases of multiple myeloma (MM) were diagnosed in 2016 in the USA, and it remained the second most common hematologic malignancy diagnosed after chronic lymphocytic leukemia [1]. Since 2015, four new drugs were approved for the treatment of relapsed/ refractory MM: panobinostat, daratumumab, ixazomib, and elotuzumab. Additionally, there were regulatory approvals for carfilzomib-based and daratumumab-based combination therapy in relapsed/refractory myeloma as well as for lenalidomide maintenance therapy after autologous transplantation. In the carfilzomib-based and daratumumab-based regulatory trials, combination therapy resulted in unprecedented complete response (CR) rates ranging 20-40\% [2-4]. However, relapse remains common and highlights the need for additional treatment options when patients progress on novel anti-myeloma therapy. These options include enrollment in a clinical trial, re-challenging with previously used agents, salvage autologous stem cell transplantation (ASCT), or salvage chemotherapy.

Several salvage high-intensity chemotherapy options have been designed to rapidly control progressive myeloma. Among the listed options in the National Comprehensive Cancer Network multiple myeloma guidelines are dexamethasone, cyclophosphamide, etoposide, and cisplatin (DCEP), bortezomib, thalidomide, dexamethasone, cisplatin, doxorubicin, cyclophosphamide, and etoposide (VTD-PACE) or another variation such as DT-PACE without bortezomib. Another older treatment option that can be considered is vincristine, carmustine (BCNU), melphalan, cyclophosphamide, and prednisone (VBMCP) [5].

VBMCP was devised as a means of improving response rates over melphalan and prednisone (MP) [6]. Melphalan has been used to treat patients with MM for over half a century [7]. Two large meta-analysis were conducted in the 1990 s to determine if there was any benefit of melphalanbased combination chemotherapy (CCT) compared to MP in patients with newly diagnosed myeloma. In the publication by Gregory et al., there was no significant

\footnotetext{
*Corresponding author: Jan S Moreb, MD., Division of Hematology and Oncology, Department of Medicine, University of Florida, 1600 SW Archer Road, Po Box 100277, Gainesville, Florida, USA. Tel.: 352-2737499; Fax: 3522735006; Email: morebjs@medicine.ufl.edu

Received 15 August 2017 Revised 20 October 2017 Accepted 26 October 2017 Published 1 November 2017

Citation: Khan AM, MuzaffarJ, Mramba LK, MorebJS. Newsalvage vincristine carmustine, cyclophosphamide, and prednisone (VBCP) chemotherapy for relapsed/refractory multiple myeloma in the modern era: A case series. J Cancer Res Ther. 2017; 5(10):61-65. DOI: 10.14312/2052-4994.2017-12

Copyright: (c) 2017 Khan AM, et al. Published by NobleResearch Publishers This is an open-access article distributed under the terms of the Creative Commons Attribution License, which permits unrestricted use, distribution and reproduction in any medium, provided the original author and source are credited.
} 
difference in overall survival between melphalan-based CCT and MP, however MP was inferior for those patients with a poor prognosis [8]. On the other hand, there was no evidence that poor-risk patients benefited more from CCT when the Myeloma Trialists' Collaborative Group conducted their meta-analysis [9]. None-the-less, large trials like the Eastern Cooperative Oncology Group (ECOG) E2479 study found VBMCP more effective than MP in producing and sustaining remission in patients with $\mathrm{MM}$ [5]. However, melphalan has significant hematologic doselimiting toxicity; the leukopenia and thrombocytopenia observed with melphalan can be delayed and prolonged, which is the reason behind giving the regimen every 35 days. Thus, we wanted to modify this regimen and avoid melphalan due its prolonged myelosuppression and its known leukemogenic and potentially mutagenic effects.

The VBMCP regimen consists of vincristine $1.2 \mathrm{mg} / \mathrm{m}^{2} \mathrm{IV}$ day 1 , carmustine (BCNU) $20 \mathrm{mg} / \mathrm{m}^{2}$ IV day 1 , melphalan $8 \mathrm{mg} / \mathrm{m}^{2}$ PO days 1-4, cyclophosphamide $400 \mathrm{mg} / \mathrm{m}^{2}$ IV day 1 , and prednisone $40 \mathrm{mg} / \mathrm{m}^{2} \mathrm{PO}$ days $1-7$, given every 35 days. In our new regimen, $\mathrm{VBCP}$, melphalan is omitted and cyclophosphamide $400 \mathrm{mg} / \mathrm{m}^{2}$ IV is given days $1-4 \mathrm{in}$ 28-35 day cycle. We report our limited experience with this salvage regimen in heavily pretreated MM patients who failed almost all the available novel and targeted therapies.

\section{Patients and methods}

\section{Patient selection}

We conducted a retrospective, IRB approved study (IRB201601388), in which case records of patients with relapsed/refractory MM treated with BCNU containing regimens at the University of Florida from January 1, 2006 onwards were reviewed. Patients were identified using institutional databases and were included if they received at least one cycle of above therapy. Pre specified clinical information was obtained from the same databases, including age, sex, date of birth, race, Karnofsky performance status (KPS), laboratory and radiologic data, bone marrow biopsy, cytogenetics/fluorescence in situ hybridization results, prior lines of therapy, time to progression, progression free survival (PFS), and overall survival (OS).

\section{Treatment and response evaluation}

Only patients who received the combination VBCP, a modification of VBMCP, were included in the analysis, while all other carmustine containing regimens such as VBMCP were excluded. Patients were treated in the outpatient infusion center. VBCP regimen includes vincristine 1.2 $\mathrm{mg} / \mathrm{m}^{2}$ (not to exceed $2.0 \mathrm{mg}$ ) IV day 1, BCNU $20 \mathrm{mg} / \mathrm{m}^{2}$ IV day 1 , cyclophosphamide $400 \mathrm{mg} / \mathrm{m}^{2}$ IV days $1-4$, and prednisone $40 \mathrm{mg} / \mathrm{m}^{2}$ days 1-7, given every 28-35 days. Growth factor support was given to all patients either with daily filgrastim or pegfilgrastim. Response was graded using the International Myeloma Working Group (IMWG) uniform response criteria [10]. Toxicity was assessed using the Cancer Terminology Criteria for Adverse Events (CTCAE), version 4.0.

\section{Statistics}

Descriptive statistics (median, range, and proportions) were used to describe the patient characteristics, response rates, and frequency of toxicities. PFS was assessed from the start of VBCP chemotherapy to the date of progression or date of death. OS was calculated to the date of death due to any cause. Patients alive without progression or living were censored on the date of last follow-up for PFS or OS, respectively. Median PFS and OS were estimated by Kaplan-Meier method. Statistical analysis was performed using SPSS Statistics software version 24 (IBM Corp, released 2016).

\section{Results}

\section{Patient characteristics}

Ten patients with a diagnosis of MM who received VBCP chemotherapy between January 1, 2006 and August 31, 2016 were identified. All patients were treated in the outpatient setting and hospitalized once developed neutropenic fever or other significant complications. Patient characteristics are listed in Table 1. The median age at

Table 1 Baseline characteristics of patients receiving VBCP chemotherapy $(\mathrm{n}=10)$.

\begin{tabular}{|c|c|}
\hline Characteristic & \\
\hline Age at diagnosis, median (range) & $63(51-73)$ \\
\hline Male, n (\%) & $5(50)$ \\
\hline Female, n (\%) & $5(50)$ \\
\hline \multicolumn{2}{|l|}{ Race, n (\%) } \\
\hline Caucasian & $5(50)$ \\
\hline African American & $5(50)$ \\
\hline \multicolumn{2}{|l|}{ ISS stage at diagnosis, n (\%) } \\
\hline I & $4(40)$ \\
\hline II & $4(40)$ \\
\hline III & $2(20)$ \\
\hline \multicolumn{2}{|l|}{ High-risk features at diagnosis, n (\%) } \\
\hline Extramedullary disease & $1(10)$ \\
\hline Extramedullary disease at time of VBCP & $2(20)$ \\
\hline High risk cytogenetics* at diagnosis & $2(20)$ \\
\hline \multicolumn{2}{|l|}{ M-spike type, n (\%) } \\
\hline $\lg G$ & $7(70)$ \\
\hline $\lg A$ & $1(10)$ \\
\hline Kappa only & $2(20)$ \\
\hline Karnofsky score, median (range) & $70(60-80)$ \\
\hline \multicolumn{2}{|l|}{ Prior therapies, $n$} \\
\hline Thalidomide & 3 \\
\hline Lenalidomide & 10 \\
\hline Pomalidomide & 6 \\
\hline Bortezomib & 10 \\
\hline Carfilzomib & 8 \\
\hline Cyclophosphamide & 9 \\
\hline Autologous stem cell transplant, single & 9 \\
\hline Autologous stem cell transplant, salvage & 3 \\
\hline Number of prior regimens, median (range) & $5.5(4-11)$ \\
\hline
\end{tabular}

*High-risk cytogenetics: del (17p), t (4;14), t (14;16) on FISH 
diagnosis of multiple myeloma was 63 with five males and five females, and an equal distribution of Caucasian and African Americans. According to the International Staging System (ISS), four patients had stage 1, four patients had stage 2 , and two had stage 3 disease at the time of initial diagnosis. Most had a good KPS at enrollment (median of $70 \%$ ). Patients were heavily pretreated with a median of 5.5 prior regimen (range, $4-11$ ). Greater than $90 \%$ of patients were previously exposed to lenalidomide, bortezomib, and an alkylating agent. Six (60\%) patients were previously treated with pomalidomide and $8(80 \%)$ patients were previously treated with carfilzomib. Nine patients (90\%) had undergone one autologous stem cell transplant (ASCT) after diagnosis and three (30\%) had salvage $2^{\text {nd }}$ ASCT, all prior to VBCP chemotherapy.

\section{Treatment characteristics and disease response}

The objective response rate (ORR, $\geq$ partial response) was $60 \%$ with an additional 30\% achieving minimal response or stable disease (Table 2). One patient had progressive disease. The median time to response for patients with at least a partial response was 1.2 months (range, 0.4 2.4 months) and the median time to progression (TTP) for patients with stable disease or better was 3.6 months (range, 0.7- > 38.9 months). The median PFS was 4.4 months $(95 \% \mathrm{Cl} 1.32-7.48)$ and the median OS was 12.8 months (95\% Cl 4.03 - 21.63) (Figure 1).

Table 2 Patients response to VBCP chemotherapy.

\begin{tabular}{|c|c|c|c|c|c|c|c|}
\hline Patient & $\begin{array}{l}\text { Time from Dx to } \\
V B C P \text { (months) }\end{array}$ & $\begin{array}{l}\text { Number of prior } \\
\text { lines of therapy }\end{array}$ & Best response & Number of cycles & TTP (months) & Next line of therapy & $A / D$ \\
\hline$A$ & 51.6 & 4 & VGPR & 3 & 8.2 & Salvage ASCT & $D$ \\
\hline C & 19.7 & 4 & MR & 1 & 0.8 & Salvage ASCT & $A$ \\
\hline D & 70.9 & 9 & PR & 5 & 2.9 & $\begin{array}{l}\text { Hospice (progressive } \\
\text { neuropathy) }\end{array}$ & $D$ \\
\hline$E$ & 117.1 & 9 & VGPR & Ongoing (> 15) & $\begin{array}{l}\text { Not reached } \\
\quad(>39)\end{array}$ & Ongoing & A \\
\hline $\mathrm{F}$ & 72.4 & 6 & VGPR & 8 & 6.7 & Daratumumab & $\mathrm{D}$ \\
\hline G & 45.7 & 5 & PR & 3 & 3.6 & Salvage ASCT & $A$ \\
\hline $\mathrm{H}$ & 75.7 & 11 & SD & 1 & 0.7 & Melphalan + Dex & $A$ \\
\hline$J$ & 23.8 & 5 & $\mathrm{PR}^{*}$ & 1 & Not reached & Daratumumab & $A$ \\
\hline
\end{tabular}

Abbreviations: A/D - alive/dead; ASCT - autologous stem cell transplantation; Dex - dexamethasone; Dx - diagnosis; MR - minimal response; PR - partial response; SD - stable disease; TTP - time to progression; Tx - Therapy; VGPR - very good partial response; VTD-PCE - bortezomib, thalidomide, dexamethasone, cisplatin, cyclophosphamide, etoposide.

*Patient had near complete resolution of innumerable subcutaneous plasmacytomas, largest was about $10 \mathrm{~cm}$ Rt submandibular mass.
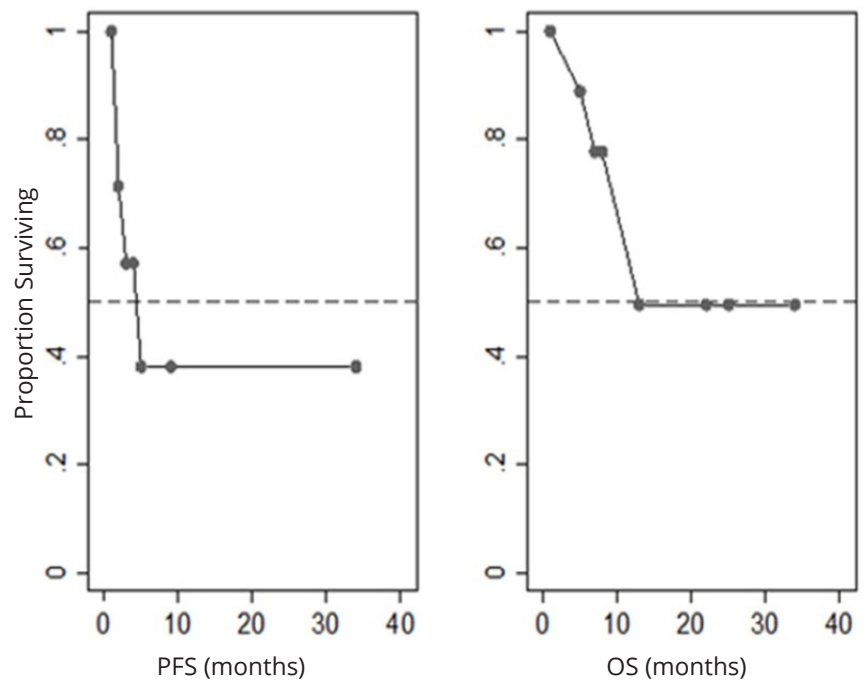

Figure 1 Kaplan-Meier curves for progression-free survival (PFS) and overall survival (OS) for patients treated with VBCP chemotherapy.

\section{Toxicities to VBCP chemotherapy}

This combination was in general well tolerated and $61 \%$ of the treatment cycles were given on time, every 28-35 days.
Delay in blood count recovery and hospitalization due to neutropenic fever were the main reasons for delaying the next cycle beyond 35 days from prior cycle. Treatmentrelated adverse events are presented in Table 3. Grade 3 and 4 hematologic toxicities were common with transfusionrequiring anemia encountered in $50 \%$ of patients and neutropenia and thrombocytopenia encountered in $60 \%$ of patients. Neutropenic fever was observed in $20 \%$ of patients. Grade 3-4 infection without neutropenia occurred in $50 \%$ of patients. Hematologic myelosuppression was the reason for reducing the cyclophosphamide dose to two days (50\%) in $20 \%$ of patients. Other non-hematologic complications of VBCP chemotherapy included electrolyte abnormalities, renal insufficiency, tumor lysis, neuropathy, vomiting, and atrial fibrillation. No treatment-related mortality (TRM) was observed.

\section{Discussion}

Vast progress has been made in the treatment of patients with MM, perhaps more than any other malignancy. The number of approved agents has rapidly grown so that treating oncologists face new challenges in the management of relapsed/refractory disease. Among those 


\begin{tabular}{|c|c|c|c|}
\hline Toxicity & Grades $1-2, \%$ & Grade 3, \% & Grade 4, \% \\
\hline Anemia & 20 & 50 & \\
\hline Thrombocytopenia & & 50 & 10 \\
\hline Neutropenia & 10 & 30 & 30 \\
\hline Neutropenic fever & & 20 & \\
\hline Upper resp infection & 10 & & \\
\hline Lung infection & & 10 & \\
\hline Skin infection & & 10 & \\
\hline Enterocolitis & & 20 & \\
\hline Infection, other & 20 & 10 & \\
\hline Vomiting & 30 & & \\
\hline Atrial fibrillation & & 10 & \\
\hline Hyponatremia & 10 & 10 & \\
\hline Hypokalemia & 20 & & \\
\hline Hypophosphatemia & & 20 & 10 \\
\hline Hypomagnesemia & 10 & & \\
\hline Acute kidney Injury & 10 & & \\
\hline Tumor lysis & & 10 & \\
\hline Neuropathy & & 20 & \\
\hline
\end{tabular}

challenges is the optimal therapy of multiply relapsed/ refractory myeloma (RRMM). Prior to the development of newer generation immunomodulatory agents, proteasome inhibitors, and monoclonal antibodies, relapsed disease was often treated with combination chemotherapy. In this study, we present results of retrospective analysis on heavily pretreated patients with RRMM who received a new VBCP salvage chemotherapy, which is modified from VBMCP regimen.

In this study, patients treated with VBCP had an ORR of $60 \%$ and an additional 30\% achieved minimal response or stable disease. These were more heavily pretreated with a median of 5.5 prior regimens. Almost all of the patients were previously treated with lenalidomide, bortezomib, carfilzomib, pomalidomide, and cyclophosphamide as well as 1-2 ASCTs. The PFS observed in these patients was 4.4 months and the median OS was 12.8 months. The short time to best response, the high ORR, but short PFS suggests that the main utility of salvage VBCP is to achieve quick control of the disease and as a bridge to novel myeloma therapy including salvage ASCT and the new approved drugs such as the monoclonal antibodies. Indeed, for $30 \%$ of the patients, VBCP was used as a bridge to salvage ASCT. One patient was subsequently treated with daratumumab after relapsing on therapy while another patient was able to be switched to daratumumab therapy as a more convenient treatment/maintenance in the outpatient. One patient was subsequently treated with pomalidomide-based therapy while two others required other salvage chemotherapy. Of interest, one patient previously treated with 9 lines of therapy has been maintained on VBCP therapy for over three years and has remained in continuous very good partial response (VGPR). In this patient, in order to minimize toxicity while continuing this effective therapy, VBCP was dose-adjusted and the frequency of administration was extended to every 8 weeks with sustained response.

VBCP effectiveness seems equivalent to other salvage chemotherapy combinations, such as VTD-PACE and
hyperCVAD, used in our practice, but with the advantage of being able to give VBCP in the outpatient setting as opposed to the other two regimens that have to be given in the inpatient setting. All these regimens are used interchangeably in our practice, however we have seen patients who failed VTD-PACE or hyperCVAD responding to VBCP. Furthermore, VBCP effectiveness also seems equivalent or better in comparison to other published salvage chemotherapy regimens and in similar MM patient population. Most patients diagnosed with myeloma in the modern era are not exposed to classic chemotherapy agents which usually has different mechanism of action than that of novel agents. Thus, the use of this drug combination later in the salvage setting may improve the survival of these patients. A number of groups have highlighted their experience with different combination salvage therapies in patients with aggressive or RRMM in the modern era. Gerrie et al. reported data on the use of dexamethasone \pm thalidomide with infusion of cisplatin, doxorubicin, cyclophosphamide, and etoposide $[D(T)$ $\mathrm{PACE}]$ in patients with a median of three prior treatment regimens [11]. In their review of data from 75 patients, the ORR was $49 \%$ while another $36 \%$ had either minimal response or stable disease. The PFS was 5.5 months and the OS was 14 months. Subsequently, another report on 51 patients highlighted the utility of dexamethasone, cyclophosphamide, etoposide, and cisplatin (DCEP) in this type of patients [12]. The ORR was $45 \%$ with the majority of patients achieving better than partial response while $33 \%$ had minimal response or stable disease. As with the prior study, patients had a median of 3 prior treatment regimens. The median PFS was 3.7 months and the median OS was 8.0 months. Finally, in a comparative study, outcomes with DCEP, VTD-PACE, and cyclophosphamide, vincristine, doxorubicin, and dexamethasone (CVAD) salvage were reported [13]. The ORR were 52\%, 73\%, and $49 \%$, respectively. These patients were treated with a median of 3 prior regimens.

Toxicity was fairly common with VBCP chemotherapy. As expected in this heavily pretreated MM patients, grade 3-4 myelosuppression was the most common toxicity. This is most likely caused by the quadrupling the cyclophosphamide dose from one day to 4 days. Twenty percent of patients had neutropenic fever while another $50 \%$ had non-neutropenic infections mainly of skin, respiratory or gastrointestinal tract. Serious noninfectious toxicity included electrolyte abnormalities, renal injury, tumor lysis, and atrial fibrillation. One patient had debilitating neuropathy and transitioned to hospice care after VBCP chemotherapy. Pulmonary toxicity was not observed.

The VBCP regimen consists of vincristine, BCNU, cyclophosphamide, and prednisone. Compared to VBMCP, the dose of cyclophosphamide is increased and melphalan is omitted. While direct comparison is not possible, VBMCP chemotherapy toxicity included leukopenia in 22 of 46 patients and combined leukopenia and thrombocytopenia in an additional 10 patients requiring dose reductions and treatment delay in patients with melphalan-resistant myeloma [14]. VBMCP induction and consolidation therapy in patients with newly diagnosed MM resulted in grade 3 or 
higher hematologic toxicity in $72 \%$ of patients in the study by Kyle et al. [15]. Our decision to omit melphalan from the regimen was based on the fear that the prolonged nadir of melphalan-induced myelosuppression will delay treatment and cause more prolonged long-term marrow toxicity. In our heavily pretreated patients managed with VBCP chemotherapy, $80 \%$ of patients had grade $\geq 3$ hematologic toxicity. Despite that, $61 \%$ of the subsequent cycles were given on time.

BCNU is one of the agents that differentiate VBCP from DCEP, VTD-PACE, and CVAD. Like cyclophosphamide, melphalan, and cisplatin, BCNU is an alkylating agent that has been used in the treatment of MM in different combinations. These published combinations include $B C N U$, doxorubicin, and prednisone (BAP) [16], vincristine, $B C N U$, doxorubicin, and prednisone or dexamethasone (VBAP/VBAD) [17-19]. In phase I/II study, BCNU (300 mg/ $\mathrm{m}^{2}$ ) was used with high-dose melphalan conditioning regimen prior to ASCT [20]. By adding etoposide and BCNU to melphalan conditioning, a CR rate post BEM-autologous stem cell transplantation was $64 \%$ with an ORR of $97 \%$ [21]. Finally, V-BEAM (bortezomib-BEAM) conditioning prior to second ASCT for RRMM produced a CR rate at day +100 of $75 \%$ [22]. However, due to unexpected treatmentrelated toxicity of infectious colitis, neutropenic colitis, and overwhelming sepsis, the study was terminated after enrolling 10 patients.

\section{Conclusions}

Although our study is limited by the small size of patients and retrospective nature, our analysis suggests VBCP is efficacious in producing an objective response with tolerable side effects in heavily pretreated population of RRMM. VBCP can be considered as a salvage option for patients who do not qualify for clinical trials, are awaiting clearance for novel anti-myeloma therapy, or have rapidly progressive disease.

\section{Conflicts of interest}

We have no conflicts of interest to disclose.

\section{References}

[1] Siegel RL, Miller KD, Jemal A. Cancer Statistics, 2017. CA Cancer J Clin. 2016; 67(1):7-30.

[2] Stewart AK, Rajkumar SV, Dimopoulos MA, Masszi T, Špička I, et al. Carfilzomib, lenalidomide, and dexamethasone for relapsed multiple myeloma. N Engl J Med. 2015; 372(2):142-152.

[3] Palumbo A, Chanan-Khan A, Weisel K, Nooka AK, Masszi T, et al. Daratumumab, bortezomib, and dexamethasone for multiple myeloma. N Engl J Med. 2016; 375(8):754-766.

[4] Dimopoulos MA, Oriol A, Nahi H, San-Miguel J, Bahlis NJ, et al. Daratumumab, lenalidomide, and dexamethasone for multiple myeloma. N Engl J Med. 2016; 375(14):1319-1331.

[5] Oken MM, Harrington DP, Abramson N, Kyle RA, Knospe W, et al. Comparison of melphalan and prednisone with vincristine, carmustine, melphalan, cyclophosphamide, and prednisone in the treatment of multiple myeloma: Results of Eastern Cooperative Oncology Group Study E2479. Cancer. 1997; 79(8):1561-1567.

[6] Lee BJ, Sahakian G, Clarkson BD, Krakoff IH. Proceedings: Combination chemotherapy of multiple myeloma with alkeran, cytoxan, vincristine, prednisone, and BCNU. Cancer. 1974; 33(2):533-538.

[7] Kyle RA, Rajkumar SV. Multiple myeloma. Blood. 2008; 111(6):29622972.
[8] Gregory WM, Richards MA, Malpas JS. Combination chemotherapy versus melphalan and prednisolone in the treatment of multiple myeloma: An overview of published trials. J Clin Oncol. 1992; 10(2):334342.

[9] Myeloma Trialists' Collaborative Group. Combination chemotherapy versus melphalan plus prednisone as treatment for multiple myeloma: An overview of 6,633 patients from 27 randomized trials. J Clin Oncol. 1998; 16(12):3832-3842.

[10] Kumar S, Paiva B, Anderson KC, Durie B, Landgren O, et al. International Myeloma Working Group consensus criteria for response and minimal residual disease assessment in multiple myeloma. Lancet Oncol. 2016; 17(8):e328-e346.

[11] Gerrie AS, Mikhael JR, Cheng L, Jiang H, Kukreti V, et al. D(T)PACE as salvage therapy for aggressive or refractory multiple myeloma. $\mathrm{Br}$ J Haematol. 2013; 161(6):802-810.

[12] Park S, Lee SJ, Jung CW, Jang JH, Kim SJ, et al. DCEP for relapsed or refractory multiple myeloma after therapy with novel agents. Ann Hematol. 2014; 93(1):99-105.

[13] Griffin PT, Ho VQ, Fulp W, Nishihori T, Shain KH, et al. A comparison of salvage infusional chemotherapy regimens for recurrent/refractory multiple myeloma. Cancer. 2015; 121(20):3622-3630.

[14] Steinke B, Busch FW, Becherer C, Ostendorf P, Waller HD. Melphalanresistant multiple myeloma: Results of treatment according to the $\mathrm{M}-2$ protocol. Cancer Chemother Pharmacol. 1985; 14(3):279-281.

[15] Kyle RA, Jacobus S, Fridenberg WR, Slabber CF, Rajkumar SV, et al. The treatment of multiple myeloma utilizing vincristine, carmustine (BCNU), melphalan, cyclophosphamide, and prednisone (VBMCP) alternating with high-dose cyclophosphamide and a2 beta-interferon versus VBMCP: Results of a phase III Eastern Cooperative Oncology Group Study E5A93. Cancer. 2009; 115(10):2155-2164.

[16] Kyle RA, Pajak TF, Henderson ES, Nawabi IU, Brunner K, et al. Multiple myeloma resistant to melphalan: treatment with doxorubicin, cyclophosphamide, carmustine (BCNU), and prednisone. Cancer Treat Rep. 1982; 66(3):451-456.

[17] Bonnet J, Alexanian R, Salmon S, Bottomley R, Amare $M$, et al. Vincristine, BCNU, doxorubicin, and prednisone (VBAP) combination in the treatment of relapsing or resistant multiple myeloma: A Southwest Oncology Group study. Cancer Treat Rep. 1982; 66(6):1267-1271.

[18] Bladé J, San Miguel J, Sanz-Sanz MA, Alcalá A, Hernández JM, et al. Treatment of melphalan-resistant multiple myeloma with vincristine, BCNU, doxorubicin, and high-dose dexamethasone (VBAD). Eur j Cancer. 1992; 29A(1):57-60.

[19] Boccadoro M, Marmont F, Tribalto M, Avvisati G, Andriani A, et al. Multiple myeloma: VMCP/VBAP alternating combination chemotherapy is not superior to melphalan and prednisone even in high-risk patients. J Clin Oncol. 1991; 9(3):444-448.

[20] Comenzo RL, Hassoun H, Kewalramani T, Klimek V, Dhodapkar M, et al. Results of a phase $1 / / \mathrm{I}$ trial adding carmustine $(300 \mathrm{mg} / \mathrm{m} 2)$ to melphalan $(200 \mathrm{mg} / \mathrm{m} 2)$ in multiple myeloma patients undergoing autologous stem cell transplantation. Leukemia. 2006; 20(2):345-349.

[21] Liu S, Mohrbacher A, Douer D, Kothary VR, Hanna L, et al. Combination therapy with BCNU/etoposide/melphalan (BEM) as conditioning regimen for autologous stem cell transplant in multiple myeloma. Blood. 2010; 116:4587.

[22] Wang TF, Fiala MA, Cashen AF, Uy GL, Abboud CN, et al. A phase II study of $\mathrm{V}$-BEAM as conditioning regimen before second auto-SCT for multiple myeloma. Bone Marrow Transplant. 2014; 49(11):1366-1370. 